\title{
MERGERS AND OBLIQUITIES IN STELLAR TRIPLES
}

\author{
Smadar NaOZ ${ }^{1,3}$ and Daniel C. Fabrycky ${ }^{2}$ \\ ${ }^{1}$ Harvard Smithsonian Center for Astrophysics, Institute for Theory and Computation, 60 Garden Street, Cambridge, MA 02138, USA; snaoz@ cfa.harvard.edu \\ ${ }^{2}$ Department of Astronomy and Astrophysics, University of Chicago, 5640 South Ellis Avenue, Chicago, IL 60637, USA \\ Received 2013 November 11; accepted 2014 August 4; published 2014 September 16
}

\begin{abstract}
Many close stellar binaries are accompanied by a faraway star. The "eccentric Kozai-Lidov" (EKL) mechanism can cause dramatic inclination and eccentricity fluctuations, resulting in tidal tightening of inner binaries of triple stars. We run a large set of Monte Carlo simulations, including the secular evolution of the orbits, general relativistic precession, and tides, and we determine the semimajor axis, eccentricity, inclination, and spin-orbit angle distributions of the final configurations. We find that the efficiency of forming tight binaries ( $\lesssim 16$ days) when taking the EKL mechanism into account is $\sim 21 \%$, and about $4 \%$ of all simulated systems ended up in a merger event. These merger events can lead to the formation of blue stragglers. Furthermore, we find that the spin-orbit angle distribution of the inner binaries carries a signature of the initial setup of the system; thus, observations can be used to disentangle close binaries' birth configuration. The resulting inner and outer final orbits' period distributions and their estimated fraction suggest that secular dynamics may be a significant channel for the formation of close binaries in triples and even blue stragglers.
\end{abstract}

Key words: binaries: close - binaries: general - blue stragglers - stars: general - stars: kinematics and dynamics

Online-only material: color figures

\section{INTRODUCTION}

Most massive stars reside in a binary configuration $(\gtrsim 70 \%$ of all OBA spectral type stars; see Raghavan et al. 2010). Furthermore, stellar binaries are responsible for diverse astrophysical phenomena, from X-ray binaries to Type Ia supernovae. However, probably many of these binaries are in fact triples. Tokovinin (1997) showed that $40 \%$ of short-period binary stars ( $<10$ days) in which the primary is a dwarf $\left(0.5-1.5 M_{\odot}\right)$ have at least one additional companion. This number contrasts with his estimate of the fraction of companions to binaries with a slightly larger period (10-100 days), which is $\sim 10 \%$. Moreover, Pribulla \& Rucinski (2006) have surveyed a sample of contact binaries and noted that among 151 contact binaries (brighter than $10 \mathrm{mag}$ ), $42 \% \pm 5 \%$ are at least triple. Therefore, triplestar systems are probably very common (e.g., Tokovinin 1997; Tokovinin et al. 2006; Eggleton et al. 2007; Griffin 2012).

Recently, Rappaport et al. (2013) estimated that the fraction of tertiaries within several AU of the close Kepler eclipsing binaries is about 20\% (see also Conroy et al. 2014). This is in agreement with previous estimates of the triples fraction. Rappaport et al. (2013) also reported the distribution of mutual inclination angle of their 39 triple-star candidates, which showed a significant peak around $40^{\circ}$, the nominal Kozai angle (see below).

From dynamical stability arguments these triple stars must be in hierarchical configurations, in which the inner binary is orbited by a third body on a much wider orbit. Many shortperiod compact binaries (such as black holes, neutron stars, and white dwarfs; Thompson 2011) are likely produced through triple evolution. Secular effects (i.e., coherent interactions on timescales long compared to the orbital period), and specifically Kozai-Lidov cycling (Kozai 1962; Lidov 1962, see below), have been proposed as a dynamical driver in the evolution of triple stars (e.g., Harrington 1969; Mazeh \& Shaham 1979; Kiseleva

\footnotetext{
3 Einstein Fellow.
}

et al. 1998; Fabrycky \& Tremaine 2007; Perets \& Fabrycky 2009; Thompson 2011; Naoz et al. 2013a; Shappee \& Thompson 2013; Pejcha et al. 2013; Perets 2014; Michaely \& Perets 2014). In addition, Kozai-Lidov cycling speeds the growth of black holes at the centers of dense star clusters and the formation of short-period binary black holes (Wen 2003; Miller \& Hamilton 2002; Blaes et al. 2002). In addition, Ivanova et al. (2010) estimated that the most efficient formation channel for black hole X-ray binaries in globular clusters may be triple-induced mass transfer in a black hole-white dwarf binary.

In early studies of high-inclination secular perturbations (Kozai 1962; Lidov 1962), it was assumed that the outer orbit is circular and that one of the inner binary members is a test (massless) particle. In this situation, the component of the inner orbit's angular momentum along the $z$-axis is conserved (where the $z$-axis is parallel to the total angular momentum), and the lowest order of the approximation, the quadrupole approximation, is valid. Following Lithwick \& Naoz (2011), we label this approximation as the "TPQ" (Test Particle Quadrupole) approximation. ${ }^{4}$ Recently, Naoz et al. (2011, 2013a) showed that relaxing these assumptions leads to qualitatively different behavior. Considering systems beyond the test particle approximation, or an eccentric orbit with a moderate semimajor axis ratio, requires the next level of approximation, called the octupole level of approximation (e.g., Harrington 1968, 1969; Ford et al. 2000; Blaes et al. 2002).

In the octupole level of approximation, the inner orbital eccentricity can reach very high values (Ford et al. 2000; Naoz et al. 2013a; Li et al. 2013; Teyssandier et al. 2014). In addition, the inner orbit inclination with respect to the total angular momentum can flip from prograde to retrograde - not even the sign of the orbital angular momentum's $z$-component is

\footnotetext{
4 Note that here we focus on the general problem with no restrictions on the masses; for the test particle approximation see Lithwick \& Naoz (2011), Katz et al. (2011), and Li et al. (2014).
} 
conserved (Naoz et al. 2011, 2013a). We refer to this process as the eccentric Kozai-Lidov (EKL) mechanism. It was shown in Naoz et al. (2013a) that the secular approximation can be used as a great tool to understand different astrophysical settings, from massive or stellar compact objects to planetary systems (for example, this has large consequences on retrograde hot Jupiters; e.g., Naoz et al. 2011).

We study the secular dynamical evolution of triple stars using the octupole level of approximation, including tidal effects (following Hut 1980; Eggleton et al. 1998) and general relativity (GR) effects for both the inner and outer orbit (Naoz et al. 2013b). ${ }^{5}$ The secular evolution of the triple stellar system was considered previously in the literature (e.g., Harrington 1968, 1969; Mazeh \& Shaham 1979; Kiseleva et al. 1998; Eggleton et al. 1998; Eggleton \& Kiseleva-Eggleton 2001; Eggleton \& Kisseleva-Eggleton 2006; Fabrycky \& Tremaine 2007). Specifically, we point out that Fabrycky \& Tremaine (2007) ran large Monte Carlo simulations for the evolution of triple stars including GR and tidal effects using the TPQ approximation. Here we show that the octupole level of approximation can result in additional behavior, where we focus on the following items: the formation of short-period binaries, the obliquity of inner binaries, merged systems, and the outcome of the outer orbit. We provide comparisons with observed catalog and known systems when possible. The octupole level of approximation can lead to very high eccentricities that can drive the two members of the inner orbit to collision (or grazing interactions). This was noted first by Ford et al. (2000) and later suggested to be important to white dwarf binaries by Thompson (2011), and here we quantify this including tidal interactions.

The merger between the two inner members due to the large eccentricities induced by the octupole level has been suggested recently as a possible mechanism to explain double-degenerate Type Ia supernovae (e.g., Thompson 2011; Hamers et al. 2013; Prodan et al. 2013). In this scenario a third body in the system induces large eccentricity that drives the inner binary to a near radial trajectory. Moving beyond the secular approximation, triple-body dynamics seems to still play a dominate role in causing the collision of two white dwarfs and result in Type Ia supernovae (e.g., Katz \& Dong 2012; Kushnir et al. 2013; Dong et al. 2014). There are at least two points that can affect this outcome. First, the triple population should have undergone EKL evolution before the white dwarf stage. Second, stellar evolution, and especially mass loss, can play an important role in the evolution of these systems (e.g., Perets \& Kratter 2012; Shappee \& Thompson 2013), as it will tend to expand the orbits, or even produce unbound objects (Veras \& Tout 2012). Here we present results relating to the first part, where we follow the secular evolution of triple stars. We find the parts of the parameter space that have already undergone EKL evolution and resulted in either a close system or systems that crossed their Roche limit. These systems have decoupled from the third object and thus will probably not be a part of the parameter space that is available for the double-degenerate scenario.

The formation of blue stragglers has also been discussed in connection to dynamics in triples. (Perets \& Fabrycky 2009) suggested that the close binaries created by Kozai cycles with tidal friction would then merge by losing their orbital angular momentum to magnetized winds. Geller et al. (2011); Leigh \&

\footnotetext{
5 Note that the $1 \mathrm{PN}$ interaction term, between the inner and outer orbit, mentioned in Naoz et al. (2013b) has negligible effect here.
}

Geller (2013), on the other hand, studied encounters of multiplebody systems in star clusters and found direct collisions to be a possible source of blue stragglers. Our work emphasizes the efficiency of collisions when the octupole-level interaction is taken into account, such that blue stragglers can result from prompt collisions, even for isolated triples.

Another aspect of the octupole level is that it results in a qualitatively different time evolution of the obliquity, the angle between the star spin axis and the binary orbit (Naoz et al. 2011, 2012). As more binary stars obliquities are being observed, for example, by the BANANA survey (Albrecht et al. 2012) and by other individual endeavors, we give specific predictions for the obliquities of inner binaries in triples. Below we compare our results with the current available observations and find them to be consistent with our secular model.

The paper is organized as follows: we begin by describing the numerical setup (Section 2). We present our results (Section 3) and focus on the effects of tidal dissipation and binary merger (Section 3.2), the mutual inclination and obliquity (Section 3.3), and specific analysis of the outer orbit configuration (Section 3.4). We discuss our results and predictions in Section 4.

\section{NUMERICAL SETUP}

We follow the numerical setup presented in Fabrycky \& Tremaine (2007). We set $m_{1}=1 M_{\odot} ; m_{2}$ was chosen by selecting the $q_{\text {in }}=m_{2} / m_{1}$ from a Gaussian distribution with mean of 0.23 and standard deviation of 0.42 (Duquennoy $\&$ Mayor 1991). Similarly, $m_{3}$ was set by choosing $q_{\text {out }}=$ $m_{3} /\left(m_{1}+m_{2}\right)$ from the same Gaussian distribution. This way enables calibration to different choices of initial mass function. As we will show, the final results only weakly depend on the mass ratio, and thus we expect that choosing different mass ratio distribution will have little effect. We denote the inclination angle of the inner (outer) orbit with respect to the total angular momentum by $i_{1}\left(i_{2}\right)$, so that the mutual inclination between the two orbits is $i_{\text {tot }}=i_{1}+i_{2}$. We draw the inner (outer) periods, $P_{\text {in }}\left(P_{\text {out }}\right)$, from the lognormal distribution of Duquennoy $\&$ Mayor (1991). Note that this period distribution represents the final periods of binaries population, rather than the initial one. Furthermore, there is not clear evidence that this distribution is the correct initial distribution for triples. We choose this distribution for self-consistency reasons, and as we will show, even in light of these caveats, comparing our results with observations and catalogs suggests that the EKL mechanism plays an important role in triples. The distribution of the inner and outer eccentricities ( $e_{1}$ and $e_{2}$, respectively) was chosen to be uniform, following Raghavan et al. (2010). This distribution is more conservative than a thermal distribution, i.e., uniform distribution yields less eccentric outer binaries, and since the EKL mechanism is more efficient for larger eccentricity, we are considering a more conservative case.

We then require that these initial conditions satisfy dynamical stability, such that we can separate the effect of long-term secular effects. The first condition is that the inner orbit is initially outside the Roche limit, lest the inner stars suffer a merger before the tertiary can act. The second condition is long-term stability of the triple, in which we follow the Mardling \& Aarseth (2001) criterion:

$$
\frac{a_{2}}{a_{1}}>2.8\left(1+\frac{m_{3}}{m_{1}+m_{2}}\right)^{2 / 5} \frac{\left(1+e_{2}\right)^{2 / 5}}{\left(1-e_{2}\right)^{6 / 5}}\left(1-\frac{0.3 i_{\text {tot }}}{180^{\circ}}\right) \text {. }
$$


A final criterion is

$$
\epsilon=\frac{a_{1}}{a_{2}} \frac{e_{2}}{1-e_{2}^{2}}<0.1
$$

where $\epsilon$ measures the relative amplitudes of the octupole and quadrupole terms in the triple's Hamiltonian. This is numerically similar to the stability criterion, Equation (1) (as shown in $\mathrm{Naoz}$ et al. 2013b). At the extreme of inequality (2), effects beyond the octupole may dominate the dynamics - Katz \& Dong (2012), Antognini et al. (2014), Antonini et al. (2014), and Bode \& Wegg (2014) have shown that the secular approximation fails for a strong perturber; however, the equations we adopt do not describe these situations. Our justification for ignoring these effects is that inequalities (1) and (2) are very similar numerically, meaning that there are few systems that are stable yet are poorly described by the octupole approximation.

We solve the octupole-level secular equations numerically following Naoz et al. (2013a). We also include GR precession for the inner and outer orbit following Naoz et al. (2013b). We are able to follow the spin vectors of both the primary and the secondary of the inner orbit stellar components (spins of both stars are set initially to 25 days). Specifically, we are interested in the angle between the angular momentum of the inner orbit and the spin of the stars (the spin-orbit angle, $\psi$ ), which was set initially on a uniform distribution for the primary while the secondary was set initially with $\psi=0^{\circ}$ (but we also investigated other configurations; see Section 3.3).

We also include tidal interactions for the inner binary evolution. The differential equations that govern the inner binary's tidal evolution were presented in Eggleton \& Kiseleva-Eggleton (2001). These equations take into account stellar distortion due to tides and rotation, with tidal dissipation based on the theory of Eggleton et al. (1998). The viscous timescale, $t_{V}$ (set to be five yr in all of our runs), is related to the quality factor $Q$ (Goldreich \& Soter 1966; Hansen 2010) by

$$
Q_{j}=\frac{4}{3} \frac{k_{L, j}}{\left(1+2 k_{L, j}\right)^{2}} \frac{G m}{R_{j}^{3}} \frac{P_{\text {in }}}{2 \pi} t_{V, j},
$$

where $j \in\{1,2\}$ for $m_{1}$ and $m_{2}$, respectively, and $k_{L}$ is the classical apsidal motion constant. We use the typical value $k_{L}=0.014$, valid for $n=3$ polytropes, when representing stars (Eggleton \& Kiseleva-Eggleton 2001; Fabrycky \& Tremaine 2007). Note that this approach simplifies the effects of tides, since one would expect that the viscous timescale would vary with the stellar mass and radius; however, the exact dependence is unknown.

The strength of the equilibrium tide recipe used here is that it is self-consistent with the secular approach used throughout this study. Furthermore, assuming polytropic stars, this recipe has only one dissipation parameter for each star. Using this description, we are able to follow the precession of the spin of the stars due to oblateness and tidal torques. Therefore, using this approximation enables a qualitative understanding of the physical effects in the system. When the stars are in a very close pericenter passage (eccentricity approaches unity), the equilibrium tide model breaks as higher orders modes in the stars may play larger roles, which can affect the dynamical evolution; however, this is beyond the scope of this paper.

The upper limit for each system's integration time in all our simulations was 10 Gyr. When the two inner stars are tidally captured, the integration becomes extremely expensive. Therefore, we adopt a stopping condition that satisfies both $e_{1} \leqslant 5 \times 10^{-5}$ and $P_{\text {in }} \leqslant 7$ days.
Table 1

Summary of the Simulations

\begin{tabular}{lccccc}
\hline \hline Name & $\begin{array}{c}\text { No. of } \\
\text { Systems }\end{array}$ & $\psi_{1, I C}$ & $\psi_{2, I C}$ & $\begin{array}{c}\text { Close } \\
\text { Binaries }\end{array}$ & $\begin{array}{r}\text { Roche } \\
\text { Limit }\end{array}$ \\
\hline EKL & 3050 & Uniform & $\psi_{2, I C}=0^{\circ}$ & $21 \%$ & $4 \%$ \\
EKL $\psi 90$ & 1141 & $\psi_{1, I C}=90^{\circ}$ & $\psi_{2, I C}=90$ & $21 \%$ & $4 \%$ \\
EKL $\psi 0$ & 1139 & $\psi_{1, I C}=0^{\circ}$ & $\psi_{2, I C}=0$ & $21 \%$ & $4 \%$ \\
TPQ & 2103 & Uniform & $\psi_{2, I C}=0^{\circ}$ & $16 \%$ & $2 \%$ \\
\hline
\end{tabular}

The EKL mechanism can cause very large eccentricity excitations for the inner orbit, implying a high probability that the stars will cross each others Roche limit. Following Eggleton (1983), we define the dimensionless number

$$
L_{\text {Roche }, i j}=0.49 \frac{\left(m_{i} / m_{j}\right)^{2 / 3}}{0.6\left(m_{i} / m_{j}\right)^{2 / 3}+\ln \left(1+\left(m_{i} / m_{j}\right)^{1 / 3}\right)},
$$

where $i, j \in\{1,2\}$. Note that this criterion is for circular orbits and does not necessarily describe the full dynamics of the system. Furthermore, this condition is only slightly (up to a factor of unity) less conservative than the simplified relation of $\sim 0.3\left(m_{i} / m_{j}\right)^{1 / 3}$. However, we use this as a qualitative criterion for our purposes.

In many of our simulations, the inner orbit reaches extremely high eccentricities. During excursions to high eccentricity, there is a competition between the increased efficiency of tides leading to the Kozai capture process (Naoz et al. 2011) and the possibility of destroying the system by crossing the Roche limit (see below for further discussion). To address the possibility of crossing the Roche limit, we set an additional stopping condition, which satisfies that if $a_{1}\left(1-e_{1}\right) L_{\mathrm{Roche}, i j}<R_{i}$, we stop the run and assume that the inner binary merged.

We also compare our results with Fabrycky \& Tremaine (2007) by running an additional set of Monte Carlo simulations while considering only the TPQ approximation. The only major difference in our setting of the TPQ runs compared to Fabrycky \& Tremaine (2007) is that the initial eccentricity distribution is uniform (Raghavan et al. 2010). Furthermore, in our initial period distribution we only allow systems that are above the Roche limit separation. The total number of systems we have run is specified in Table 1.

\section{RESULTS}

\subsection{The Inner Binary Period Distribution}

Similarly to Fabrycky \& Tremaine (2007), we find that the final inner orbital period's distribution is consistent with a bimodal distribution with a peak around $\sim 3$ days, as shown in Figure 1. We define "close binaries" as systems with final period shorter than 16 days; this period roughly separates the two major peaks. We also show in the bottom panel of this figure the observed period distribution of inner binaries in triples adopted from the Tokovinin (2008) public catalog (where we have scaled the theoretical distribution to the catalog's to guide the eye). The inset in the bottom panel shows the cumulative distribution of the simulated runs, as well as the observed data. The observed systems in the catalog have a typical inner binary eccentricity of about 0.5 , (Tokovinin 2008). Taking this at face value, which may point to some selection effect in the catalog, we have compared the observed cumulative distribution with our calculated distribution, limiting our final inner orbital eccentricity, $e_{1, F}<0.5$ (green solid line). This yields better 


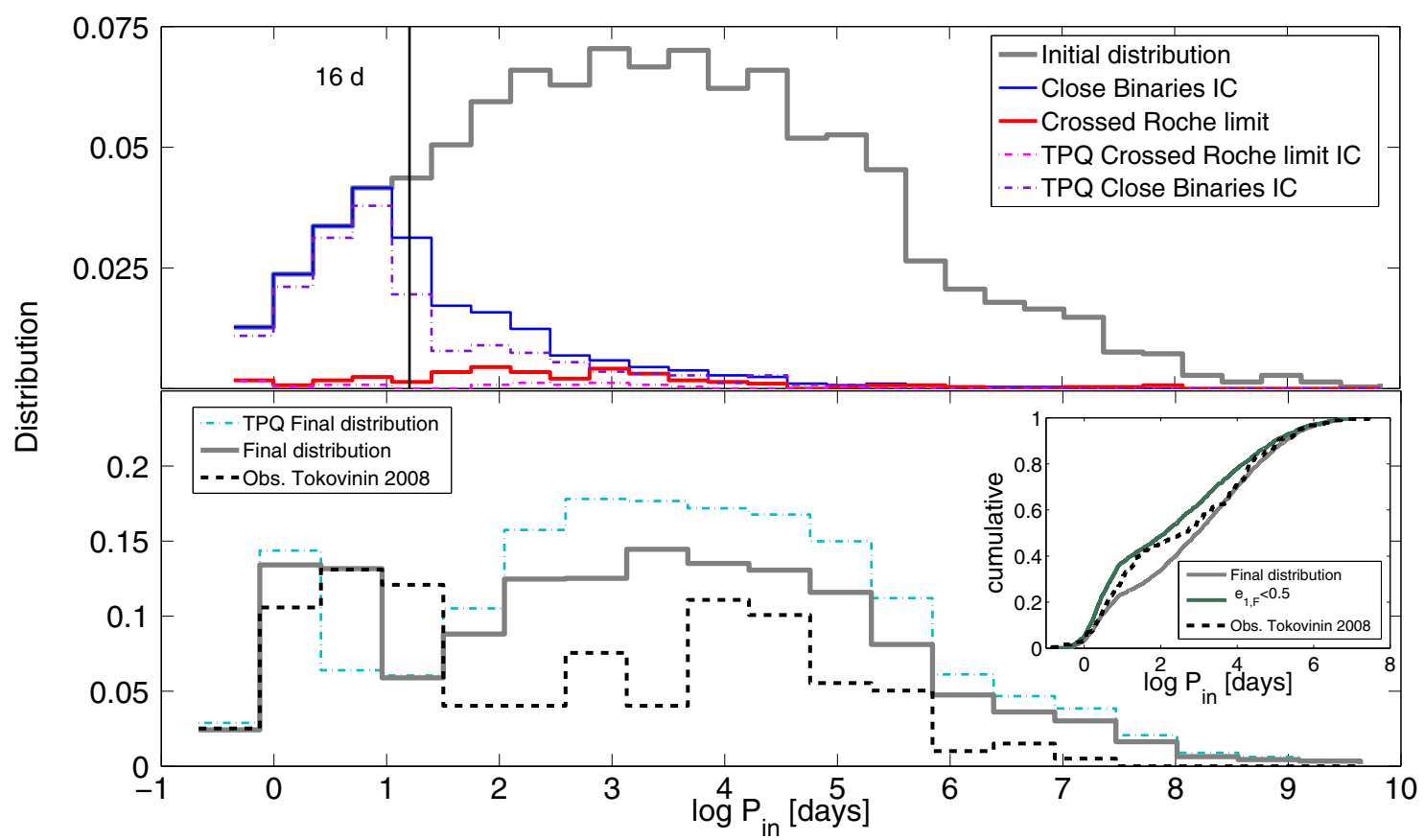

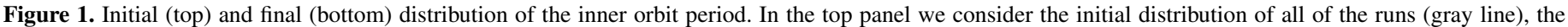

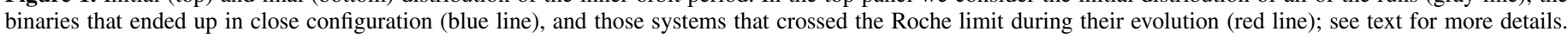

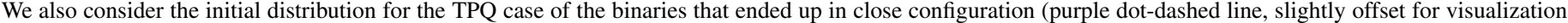

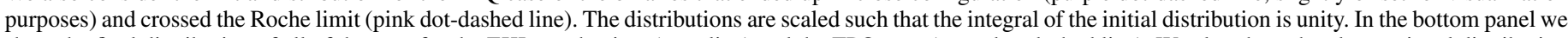

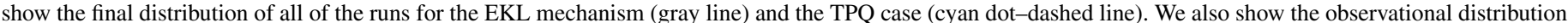

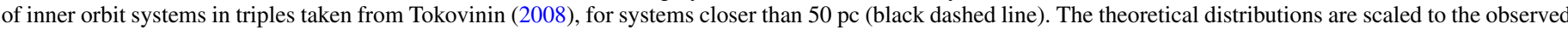

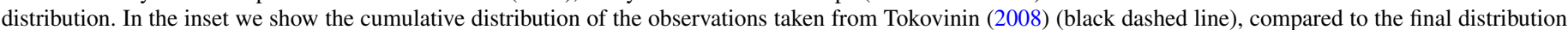

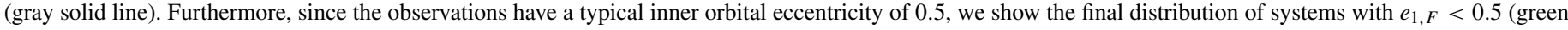
solid line).

(A color version of this figure is available in the online journal.)

agreement between the simulated period distribution and the one taken from the catalog. We note that near the completion of this paper Tokovinin $(2014 \mathrm{a}, 2014 \mathrm{~b})$ reported a new database of triple stars; here we use his old database, which mainly differs in sample size.

Interestingly, the observed bimodal distribution is reproduced with our secular evolution model. We find that the Kolmogorov-Smirnov test does not reject the null hypothesis that the observed inner orbit's distribution and the simulated one are from the same continuous distribution (where we consider the full EKL distribution, and not the eccentricity-limited one), with a $p$ value of 0.3828 . This behavior suggests that secular evolution in triples plays an important role in shaping the distribution of these systems. However, we point out two major differences between the theoretical predictions and the observational data.

The first is the period that separates the two major peaks in the distribution. The observed period distribution (adopted from the Tokovinin (2008) public catalog) has a wide valley in the distribution, with periods ranging between 25 and 100 days, while the theoretical predictions give a narrow valley in the period distribution ranging between 16 and 40 days. The explanation may lay either on the initial conditions, where we assumed that hierarchical triple period distribution follows binary population, or in our model. Here we restricted ourselves to the hierarchal three-body approximation, which means that systems that could have formed through short-timescale, scattering types of interactions are not modeled. Wide inner orbits in triple configurations have been found in scattering-like interactions between stars in an open stellar cluster in the recent work by
Geller et al. (2013), where we deduce, from their Figure 9, a minimum in the period distribution that extended to $\sim 100$ days (note that their second, long-period peak in the distribution is not as dominant as in our case for the triple population). An additional process that can produce wide inner binaries is mass loss (Perets \& Kratter 2012; Geller et al. 2013). With our model we do not capture these possible processes that can account for wider inner binaries; therefore, even in the absence of these processes, the agreement between the observed and modeled distributions suggests that secular evolution plays a dominant role for triple systems.

The above difference in the period distribution valley between the observation and the TPQ calculation in Fabrycky \& Tremaine (2007) was noted by Tokovinin (2008). Our new calculation including the octupole evolution improves on that and extends the inner period distribution peak to $\sim 16$ days, where we addressed possible causes for the differences above. However, we are encouraged that the theoretical inner orbital period distribution appears rather flat, as found in Tokovinin \& Smekhov (2002) radial velocity observations.

The second discrepancy between the observations and the theoretical predictions is in the ratio between the two peaks; in other words, at face value, there are more close inner binaries than wide ones in the catalog of the observed systems compared to the simulated triples. This can be explained as a combination of both theoretical and observational shortcomings. Here again, stellar evolution may play a role in determining the final separation of triple configurations, and may even ionize/unbind the outer orbit, as shown in Perets \& Kratter (2012) and Geller et al. (2013). As shown in the top panel of Figure 1, there is a 
large tail of wide inner binaries that can result in close binaries. Owing to the hierarchical criterion, their tertiary has an even wider orbit. The capture into a close binary due to the EKL mechanism can happen on a short timescale (as can be seen in Figure 8, top left panel), which leaves enough time for flyby perturbation or mass loss to unbind the outer orbit. This is also supported by Tokovinin et al. (2006) observations of tight binaries without a tertiary. This may imply that, in our model, we overestimate the number of wide binaries (since they are more likely to unbind).

On the other hand, wide inner binaries are harder to observe, and thus the catalog of the observed systems is incomplete and may suffer from some selection effects (A. Tokovinin 2014, private communication). The latter may be supported by the comparison with the $e_{1, F}<0.5$ final distribution (inset of Figure 1). These systems better agree with the observed distribution, which has typical inner eccentricity of 0.5 as noted in Tokovinin (2008) and Tokovinin \& Smekhov (2002). This suggests either that we are missing a piece of the physics or that observations are biased against high eccentric systems. Although this conclusion does not provide a definite answer, it points toward a possible explanation.

The similarity of the period's distribution with Fabrycky \& Tremaine (2007) is not surprising since the bulk behavior did not change by solving the equation of motions up to the octupole level of approximation. This means that both the TPQ and the EKL mechanisms produce a double-peak final period distribution; see Figure 1. However, in the presence of the octupole level, larger parts of the phase space are accessible for large inclination and eccentricity oscillations. Most importantly, the inner orbit's eccentricity may reach much larger values than the values reached with the quadrupole approximation. Therefore, wider inner binaries (compared to the TPQ approximation) and even lower inclination systems can end up forming close binaries or even drive the members of the inner orbit to merge (see Figure 1 for specific comparisons), which affects the fraction of final close and merged systems (see Table 1).

Another difference between the EKL and the TPQ approximations is the location of the minimum in the period distribution. For the TPQ this is located between $\sim 3$ and 31 days, while the EKL mechanism yields wider periods with a range between $\sim 8$ and 31 days. This is because in the EKL mechanism, the maximum eccentricity value can vary rapidly and can reach extremely large values. A system that reached a large eccentricity value can result in shrinking $a_{1}$ to some other, lower value, in a steplike way (see, e.g., Figure 2 in Naoz et al. 2011). This new value, associated with a lower $\epsilon$ value, may be less favorable for another eccentricity spike, resulting in a stable configuration on a wide inner orbit. On the other hand, the TPQ mechanism produces always the same eccentricity value, which, if high enough, can cause $a_{1}$ to shrink in a smooth way (see, e.g., Figure 1 in Fabrycky \& Tremaine 2007).

\subsection{Binary Merger and Tidal Dissipation}

During the system evolution, the octupole level of approximation can cause large eccentricity excitations for the inner orbit. Thus, on one hand the nearly radial motion of the binary drives the inner binary to merge, while on the other hand the tidal forces tend to shrink the separation and circularize the orbit. If during the evolution the tidal precession timescale (or the GR timescale) is similar to that of the Kozai timescale, further eccentricity excitations are suppressed (this was already noted in Holman et al. 1997; Fabrycky \& Tremaine 2007, for the

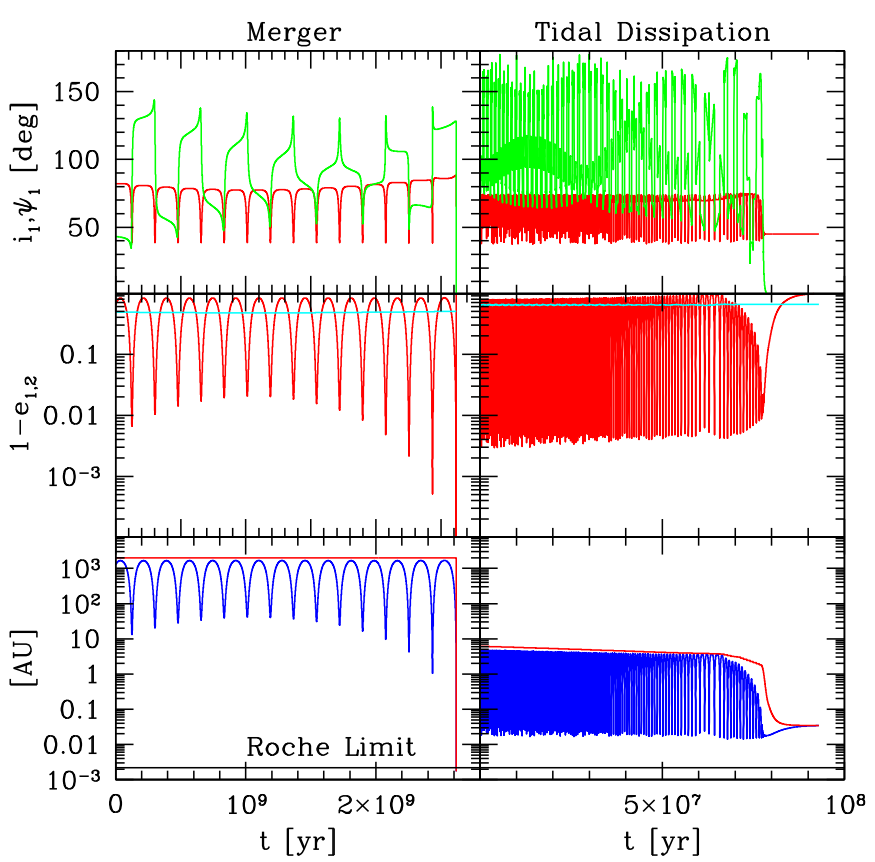

Figure 2. We show two examples chosen from the Monte Carlo runs. The left column shows a system that resulted in merger, while the right column is of an inner binary that shrinked its separation to a stable tight configuration. In the top panel we show the inclination of the inner binary with respect to the total angular momentum $i_{1}$ (red lines) and the spin-orbit angle of the primary (green angle). The middle panel shows the eccentricity as $1-e$ for the inner binary (red lines) and the outer binary (cyan lines). The bottom panel shows the inner binary separation (red lines) and the pericenter distance (blue lines). We also show the Roche limit value (black lines), which is simply $L_{\text {Roche, } 12} \times R_{1}$. The right column was set initially with $m_{2}=0.337 M_{\odot}, m_{3}=0.094 M_{\odot}$, $a_{1}=6.16 \mathrm{AU}, a_{2}=106.155 \mathrm{AU}, e_{1}=0.539, e_{2}=0.368, \omega_{1}=223.54$, $\omega_{2}=212.863$, and $i=103.02$, which means that $i_{1}=68.52$; and the system in the left column was set initially with $m_{2}=0.31 M_{\odot}, m_{3}=0.733 M_{\odot}$, $a_{1}=2001.67 \mathrm{AU}, a_{2}=31571.32 \mathrm{AU}, e_{1}=0.356, e_{2}=0.51, \omega_{1}=145.99$, $\omega_{2}=65.82$, and $i=88.41$, which means that $i_{1}=82.14$.

(A color version of this figure is available in the online journal.)

quadrupole approximation). ${ }^{6}$ In that case tides can shrink the binary separation and form a tight binary decoupled from the tertiary companion. The final binary remained in a stable orbit (note that tides always tend to shrink the binary separation, but this happens on a much longer timescale). However, if the eccentricity is excited on a much shorter timescale than the typical extra precession timescale (such as tides, rotational bulge, and the GR precession timescales, but of course still long enough so that the secular approximation is valid), the orbit becomes almost radial, and the stars can cross the Roche limit. In that case, the extra precession does not have enough time to affect the evolution. This is the process that causes, for example, tidal migrations of planets in stellar binaries until they tidally disrupt or merge (Naoz et al. 2012). In our Monte Carlo runs about $4 \%$ of the systems crossed their Roche limit and about $21 \%$ are on a tight ( $<16$ day) orbit. Hereafter we label all systems with inner orbit configuration with final period binary $<16$ days as "close binaries."

In Figure 2 we show two representative examples of the evolution of two systems that have undergone dramatic changes during their evolution due to close pericenter passages. In general, if the eccentricity excitation is evolving gradually

\footnotetext{
6 Note that when GR timescales are similar to the quadrupole level of approximation timescale, a resonant-like behavior can occur (e.g., Naoz et al. 2013b).
} 

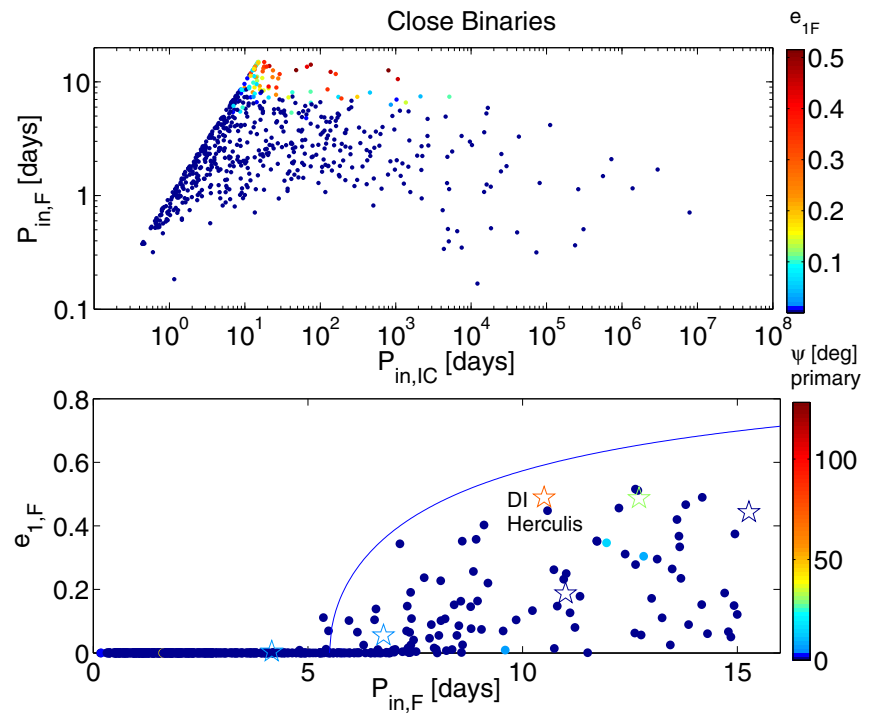

Figure 3. Close binary configuration (we consider only the close-in binaries, $P<16$ days). Top panel: final inner binary periods as a function of the initial one. The different colors mark the final eccentricity of the system. Bottom panel: we show the final eccentricity achieved as a function of the final period. The color code is the final spin-orbit angle of the primary, where most of the systems have final spin-orbit lower than $10^{\circ}$ (see Figure 6 for complementary presentation of this parameter space). Note that we show here only the primary's obliquity. The solid line represents a constant angular momentum curve with a final binary period of 5.5 days. We also plot the observations (star symbols) (Albrecht et al. 2009, 2011, 2013; Triaud et al. 2013; Harding et al. 2013; Zhou \& Huang 2013). Note that the horizontal axis in the bottom panel is in linear scale and for the top panel is in log scale.

(A color version of this figure is available in the online journal.)

(though still to larger values than achieved with the quadrupolelevel approximation), the pericenter distance shrinks slowly and allows tides to work (as shown in the example in the right column). However, during the evolution shown in the left column of Figure 2, the pericenter distance due to the EKL evolution changes by many orders of magnitude from one quadrupole timescale to the next. Thus, the angular momentum of the inner orbit is decreasing by more than an order of magnitude. The separation shrinks dramatically on a short timescale, though still larger than the inner orbit period (more than a factor of 10), and the eccentricity is decreasing on that timescale too. This happens when the inner orbit reaches large eccentricity while still on a wide separation, in which case the tidal forces (and the GR precession) do not have the time to stabilize the system and the binary components crossed each others Roche limit.

In the top panel of Figure 1 we show the initial inner orbit period distribution of the systems that merged during the evolution, as well as those that ended up in close configurations ( $<16$ days). As shown in this figure, the different outcomes (i.e., close binaries or merger) originate from two distinct populations. On average, merged binaries (marked in red) are more likely to originate from initially wider inner binaries (we discuss the outer orbit configurations for these systems in Section 3.4).

In Figure 3, top panel, we consider the relation between initial and final period of the inner close binaries' population ( $<16$ days). As shown in this figure (and also in Figure 1), the main contribution of the close binaries' population (associated with the peak in the period distribution of $\sim 3$ days) comes from systems with inner binaries with periods of $\sim 4-16$ days.

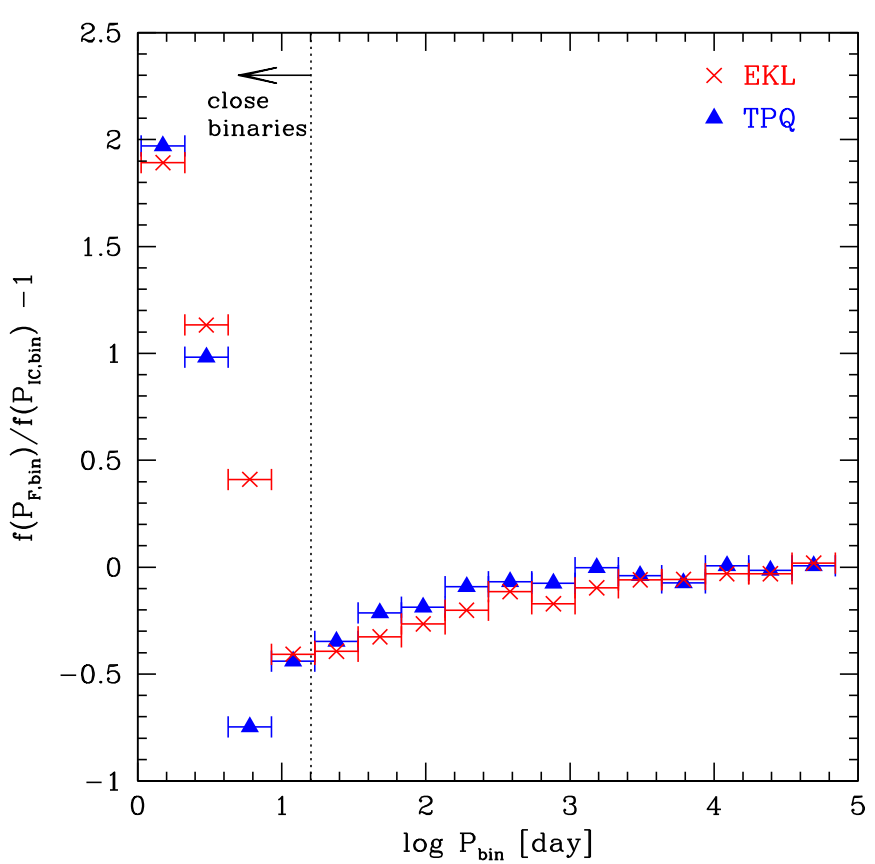

Figure 4. Fraction of systems that ended up in an inner period bin $P_{F}$,bin relative to the initial fraction of systems in that bin. We compare between the EKL (red crosses) and TPQ (blue triangles).

(A color version of this figure is available in the online journal.)

However, $\sim 41 \%$ of the close binaries originated from initial inner binary separation larger than 16 days. Considering the entire triple population, we find that about $8.6 \%$ of all triples with initially $P_{\text {in }}>16$ days have become close binaries (i.e., with $P_{\text {in, } F}<16$ days). Comparing this number with the TPQ's of $\sim 3.6 \%$, we find that the EKL efficiency is larger by about 2.4 compared to the TPQ. To illustrate further the difference between the EKL and TPQ, we consider in Figure 4 the fraction of systems in a final inner period bin relative to the initial systems in that bin (for equal logarithmic close binaries bins). The period valley in the distribution is indicative of the different efficiency of the EKL and TPQ approximations.

The population of close binaries with a wider final separation has a nonnegligible eccentricity and is in the process of tidal shrinking and circularization, as shown in the bottom panel of Figure 3. Interestingly, this bottom panel is qualitatively similar to Figure 2 in Tokovinin \& Smekhov (2002). The tidal shrinking process is still on its way even after $10 \mathrm{Gyr}$ of integration time, and those systems lay under the constant angular momentum curve (see also Figure 8, top left panel, which shows the integration time). In Section 3.4 we discuss the outer orbit configuration for those close systems.

\subsection{Inclination and Spin-orbit Angle}

The statistical distribution of mutual orbital inclinations and the spin-orbit angle can help disentangle between different formation scenarios. If the formation involves a chaotic process, one may expect an isotropic distribution of mutual inclinations of hierarchal triples. This assumption means that the third-body formation is essentially uncorrelated with the inner orbit. Our numerical experiments assumed an isotropic distribution for the initial inclination angle (i.e., uniform in $\cos i$ ).

We find, similarly to Fabrycky \& Tremaine (2007), that the initial inclination distribution is not conserved during the secular evolution. We illustrate this in Figure 5, left panel, 


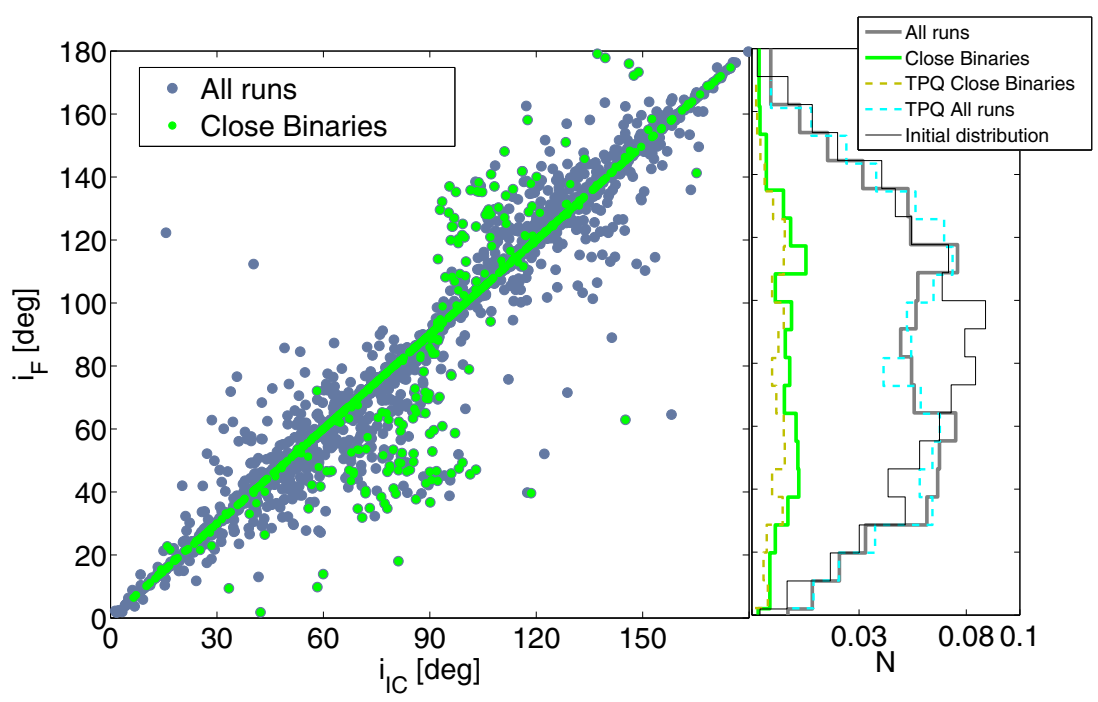

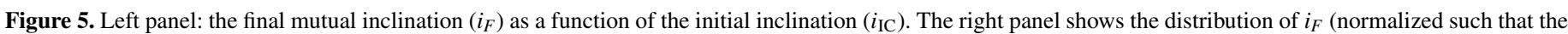

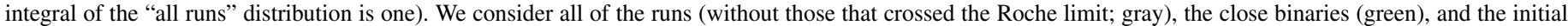

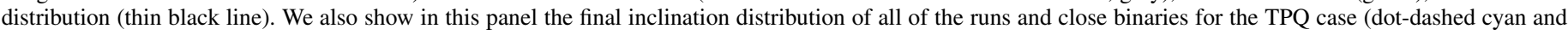
dark green, respectively).

(A color version of this figure is available in the online journal.)

where we show the final mutual inclination distribution as a function of the initial inclination. However, unlike the TPQ results presented in Fabrycky \& Tremaine (2007), their Figure 7, the final inclinations are not confined to the initial prograde or retrograde configurations, but instead are scattered in the phase space (Figure 5, left panel). This is because the EKL mechanism allows the orbits to flip from $i<90^{\circ}$ to $i>90^{\circ}$ and vice versa (Naoz et al. 2011, 2013a).

The final inclination distribution is shown in the right panel of Figure 5 for the close binaries and for all of the runs (for both the EKL and TPQ cases). Teyssandier et al. (2014) showed that in the absence of dissipation and for initial circular inner orbit, the final distribution of hierarchical triple mutual inclination has in fact three peaks, at $40^{\circ}, 90^{\circ}$, and $140^{\circ}$. The significance of these peaks depends on the initial conditions, and low eccentric outer orbits give rise to another peak at $90^{\circ}$, where the $90^{\circ}$ is more dominant; see their Figure 14, top panels. The systems that reached $90^{\circ}$ are typically associated with large eccentricity excursions and thus are more likely to have undergone tidal evolution. Thus, this peak is absent in the present of dissipation. In other words, the distribution near polar configurations is slightly more diluted, which can have large implications for the evolution of compact objects after stellar evolution that requires nearly perpendicular orbits.

The distribution show in Figure 5 yields less prominent peaks at $40^{\circ}$ and $140^{\circ}$, as predicted before. This is a result of two main reasons: (1) relaxing the test particle approximation and (2) using the EKL approximation. However, we note that, as shown in Naoz et al. (2012), a test particle (such as a hot Jupiter) set initially on a circular orbit the final inclination distribution similar to the one predicted by Fabrycky \& Tremaine (2007). Thus, the distribution of the two main peaks at $40^{\circ}$ and $140^{\circ}$ is recovered by the test particle case for the octupole level of approximation (although with slightly less significance).

Another interesting observable (and perhaps more promising than mutual inclination observations) is the spin-orbit angle (obliquity), which is the angle between the spin axis of the star and the angular momentum of the inner orbit. During the tidal evolution, the obliquity of the closest binary will most likely decay to zero. The obliquity decays faster than the eccentricity. This process produces inner binaries that are still in the process of shrinking and circularizing with typically low obliquities, as depicted in the bottom panel of Figure 3, where most of the simulated binaries below the constant angular momentum run have small obliquities $\left(\psi<50^{\circ}\right.$ for the primary and $\psi<100^{\circ}$ for the secondary; see below for more details). This behavior is also apparent in the top panel of Figure 6, where we show the final obliquity distribution. The inner binaries with an intermediate period ( $\sim 100$ days) have large eccentricities and low obliquities.

We draw attention to the top two panels of Figure 6, where there are nine close $\left(P_{\text {in }}<10\right.$ days $)$ circular systems with nonnegligible obliquity $\left(>10^{\circ}\right)$. All but one of these systems have the primary spin period matching the orbital period, but only three of the nine have secondaries that are similarly synchronized. The others are spinning considerably slower. About half of these have experienced chaotic EKL evolution, such that the outcome is a sensitive function of the initial conditions. We show the spin period of these systems for both the primary and secondary in Figure 7 . We also calculate the expected spin period from Fabrycky et al. (2007) :

$$
\Omega=2 \frac{2 \pi / P_{\text {in }}}{\cos \psi+\sec \psi},
$$

which yields that tilted systems will have smaller spin periods (see also Levrard et al. 2007). We show in Figure 7 that the expected spin period from this calculation agrees well with the results from the Monte Carlo run for the primary and the secondary.

Very recently, Albrecht et al. (2014) have reported a system, $\mathrm{CV}$ Velorum, with large obliquity $\left(52^{\circ} \pm 6^{\circ}\right.$ for the primary and $3^{\circ} \pm 7^{\circ}$ for the secondary). In the top panels of Figure 6 , this system is located near the other simulated systems that have very short periods and large obliquities. This may suggest that $\mathrm{CV}$ Velorum is a result of three-body evolution. Furthermore, the rotation period of the two stars in this system is similar to the orbital period of about seven days. This implies that, 

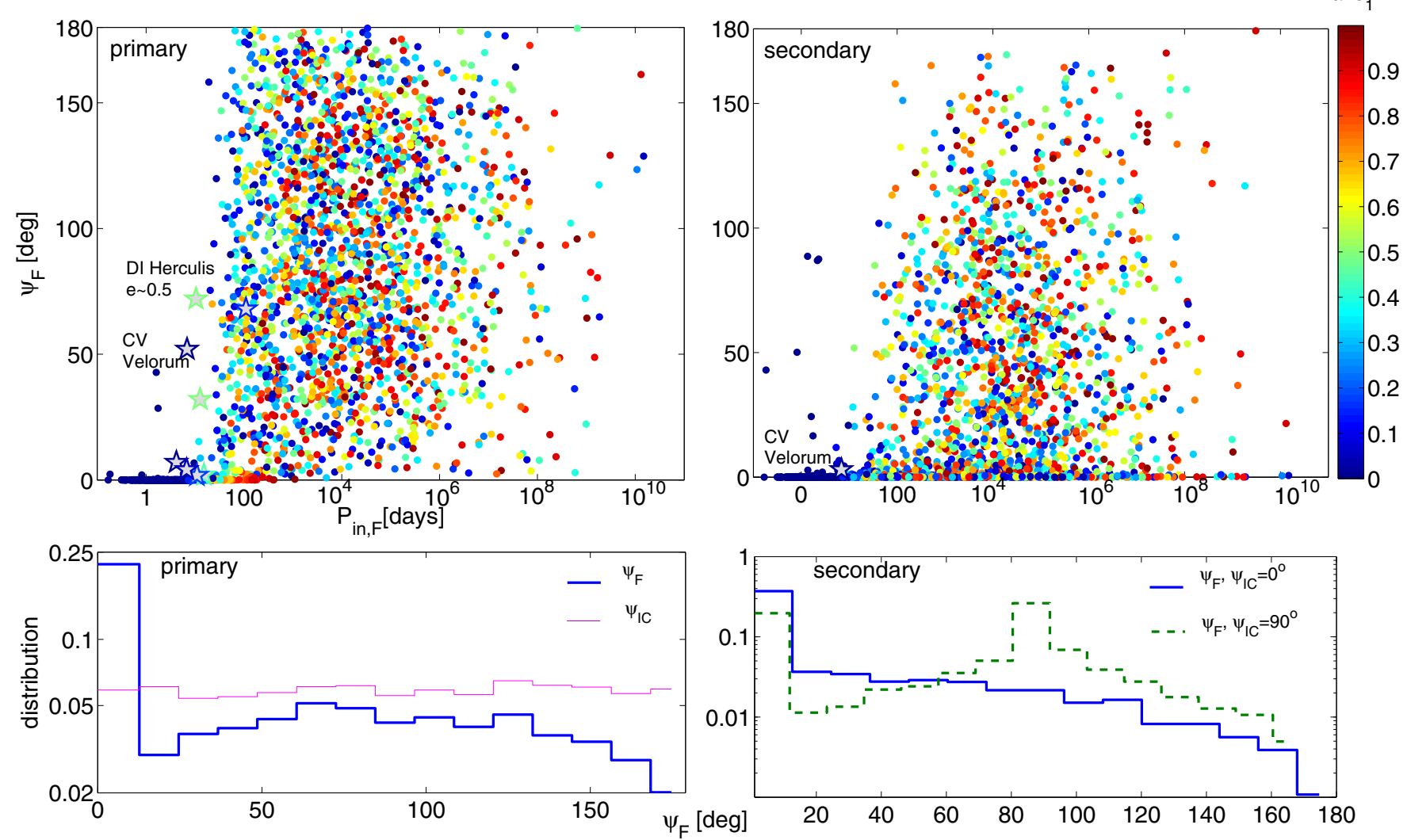

Figure 6. Spin-orbit distribution of the inner binary. Top panels: final distribution of the spin-orbit angle of the primary (left panel) and secondary (right panel) vs. the final inner orbit periods; the color code is the final eccentricity of the inner binary. We also plot the observations (Albrecht et al. 2009, 2011, 2013, 2014; Triaud et al. 2013; Harding et al. 2013; Zhou \& Huang 2013). Bottom panels: distribution of the spin-orbit angle, $\psi$ (blue lines), for the inner orbit's primary (left) and secondary (right) members. The initial distribution of $\psi$ for the primary was uniform (magenta line in the left panel), while the spin-orbit angle of the secondary was set to zero initially. Note that we have repeated the Monte Carlo runs, setting initially the primary spin-orbit angle to be aligned just like the secondary, and confirmed that in that case the final distribution is identical to the secondary one (omitted here to avoid clutter). Furthermore, to explore the effect of the initial conditions, we have had another Monte Carlo run setting initially the two spin-orbit angles on a perpendicular configuration $\left(\psi=90^{\circ}\right)$. For this case we again found that the primary and secondary final spin-orbit distributions are identical, as shown in the right bottom panel (green dashed line). Note that both vertical axes in the bottom panels are in logarithmic scale.

(A color version of this figure is available in the online journal.)

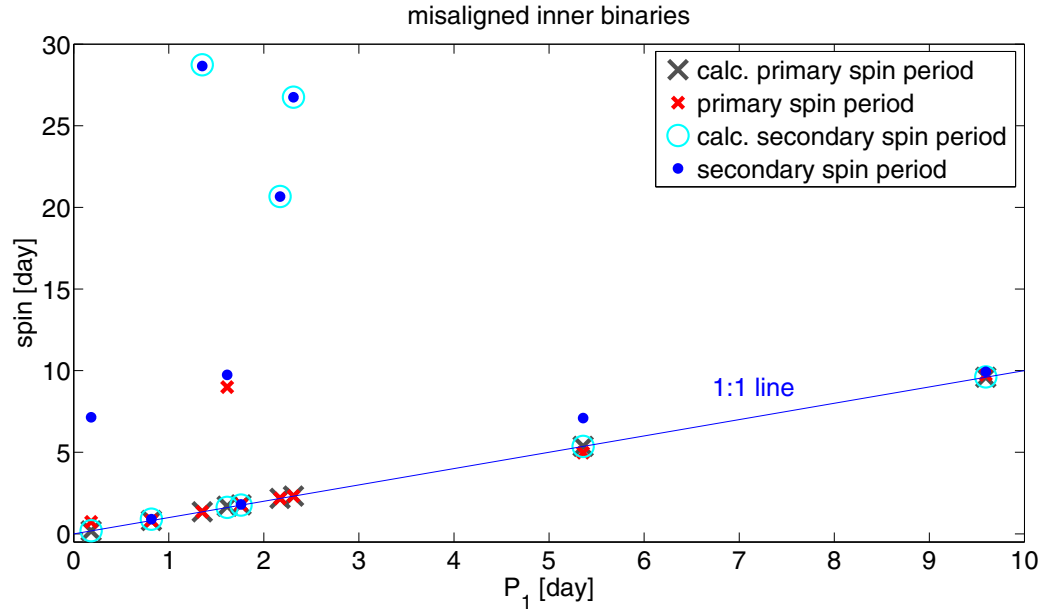

Figure 7. Misaligned close inner binaries from Figure 6. We show the simulation results for the spin periods of the primary (small red crosses) and secondary (small blue dots) as a function of period, for those close ( $P_{\text {in }}<10$ days) systems that have large obliquity $\left(>10^{\circ}\right)$. We also calculate the expected spin period from Fabrycky et al. (2007), for the primary (large gray crosses) and secondary (open cyan circles).

(A color version of this figure is available in the online journal.)

using Equation (5), one can use the rotation period to constrain the obliquity (or vice versa). For example, the $3^{\circ}$ value for the secondary obliquity agrees well with having a secondary spin period equal to the orbital period (the $1: 1$ line in
Figure 7). However, for the primary we find that a lower obliquity than the mean value (closer to the lower limit, i.e., $46^{\circ}$ ) yields an agreement between the calculated spin period and the observed one. 
An important parameter is the initial value of the obliquity. One may expect that tight binaries may be well aligned since they originate from the same portion of a molecular cloud. On the other hand, since the formation scenario of even tight binaries involves many chaotic processes, the knowledge of birth obliquity may be unknown. Therefore, we have chosen the following experiments: in the first numerical runs we set initially the primary obliquity from a uniform distribution (uniform in $\psi)$, while the secondary was chosen to be aligned with the inner orbital angular momentum (i.e., $\psi=0^{\circ}$ ). This way we can examine two different initial conditions in the same run. In the second Monte Carlo test we have set initially the two inner orbit members on a perpendicular configuration (i.e., $\psi=90^{\circ}$ ). As a consistency test, we have also had a third Monte Carlo run setting initially both the primary and the secondary on an aligned configuration and confirmed that the final obliquity distributions are identical to the secondary final distribution from the first test. Note that all the rest of the results shown in this paper are independent of the initial choice of the obliquity. For consistency reasons the other orbital parameters discussed and analyzed in the paper belong to the result from the first Monte Carlo test.

Different initial obliquity distributions are distinctive since the final distribution carries a signature for the initial setup distribution. However, another subtle difference arises in the location of the "edge" of the aligned systems, i.e., the smallest period for which most of the systems are aligned. For example, the outcome of the initial perpendicular obliquity case is that large obliquity systems can extend to very small periods with an approximate limit at $\sim 10$ days, while for the initial zero obliquity case, final aligned systems can be found for $\lesssim 40$ days.

We found that the final obliquity distribution carries a clear signature of the initial distribution. The final distribution consists of an aligned population for the tighter binaries, and a distribution that contains information of the initial setting between 0 and 40 days. This is most apparent for the second Monte Carlo test (where the $m_{1}$ and $m_{2}$ obliquities were initially set to be $90^{\circ}$ ). The final distribution has an aligned component, a broad component of obliquity up to $180^{\circ}$, but it also retained a peak at $90^{\circ}$; see the bottom right panel of Figure 6 . Setting the obliquity initially on an aligned configuration (for either the primary or the secondary) results in a final distribution of an aligned peak with a broad tail of obliquities (see bottom right panel).

There is a slight difference in setting initially $m_{1}$ and $m_{2}$ on an aligned (perpendicular) configuration compared to setting $m_{1}$ on a uniform distribution and keeping $m_{2}$ aligned (the second run). Apart from having different final obliquity distributions for the two cases (as depicted in the bottom panels of Figure 6), the "edge" of systems on a short period with low obliquities is slightly pushed inward for the case of $m_{1}$ initially aligned, compared to the uniform $m_{1}$. This can be seen by comparing $m_{1}$ and $m_{2}$ obliquities for the population with intermediate periods ( 10-100 days) and nonnegligible eccentricity; see the two top panels of Figure 6. In other words, those systems (at periods of 10-100 days with moderate to high eccentricity and low obliquities) may end up with larger obliquities when initially set on an aligned configuration (the "blue stripe" of systems in the top left panel, which is absent in the top right panel). This is easily understood if we consider the influence of the inclination oscillations due to the Kozai mechanism. During this evolution, the inner and outer argument of periapsis sweeps across $\sim 180^{\circ}$, which causes large-amplitude oscillations on the obliquity as well (see Li et al. 2013). Starting with zero obliquity thus can cause larger-amplitude oscillations and slightly suppress its damping (because of the larger torque). It is interesting to note that DI Herculis (Albrecht et al. 2009) resides in this "edge" of intermediate periods (see top left panel of Figure 6). This suggests that DI Herculis misalignment may actually be typical.

\subsection{The Outer Orbit}

The outer orbit gravitational perturbations can cause large eccentricity oscillations for the inner orbit, as discussed above. Strong perturbations can result in shrinking of the inner orbit separation or even lead to merger of the inner members (see Section 3.2 for details regarding the inner orbit's properties). The outer orbit configuration sets the different outcomes of the inner orbit, and thus a promising observable is the outer orbit's period distribution. In the bottom right panel of Figure 8 we show the initial outer orbit period taken from the lognormal distribution of Duquennoy \& Mayor (1991). We also show the period distribution of the population of outer orbits that produced close inner binaries (blue line). Interestingly, this distribution is completely different from the injected initial distribution. We also show in this figure the observed outer orbit's distribution (adopted from Tokovinin 2008, public catalog), which we scaled to guide the eye. Note that the observed distribution is not limited to the close binaries. However, probably due to observational limitations in compiling the catalog, we suspect that it will be biased toward companions that are around the close binary population. Thus, it is not surprising that the Kolmogorov-Smirnov test does not reject the null hypothesis at $5 \%$ significance level that the observed outer orbit's distribution and the simulated one are from the same continuous distribution, with $p$ value of 0.1564 . Therefore, although we have a long tail of wide outer orbits, close binaries (with periods $\lesssim 16$ days) have preferentially wide outer orbits, with a peak distribution at $\sim 10^{6}$ day (as shown in Figure 8 bottom right panel), in agreement with Tokovinin (2008) Figure 3.

The close inner binary's final separation represents the final stage of the secular evolution in the presence of tides and GR, where the outer orbit's separation does not change. When the EKL precession timescale is comparable to tidal (and GR) precession, further eccentricity excitations from the EKL evolution are suppressed. The inner orbit then settles on the separation that equalized these timescales, or shorter separations. The timescale associated with the Newtonian quadrupole term, due to the outer body, can be estimated from the canonical equations (e.g., Naoz et al. 2013a)

$$
t_{\text {quad }} \sim \frac{2 \pi a_{2}^{3}\left(1-e_{2}^{2}\right)^{3 / 2} \sqrt{m_{1}+m_{2}}}{a_{1}^{3 / 2} m_{3} \sqrt{G}}
$$

where $G$ is the gravitational constant. The tidal precession timescale (ignoring the spin term) is estimated as (e.g., Eggleton et al. 1998)

$$
t_{\mathrm{TF}} \sim \frac{a_{1}^{13 / 2}\left(1-e_{1}^{2}\right)^{5} m_{1} m_{2}}{15 \sqrt{G} \sqrt{m_{1}+m_{2}} f_{e} \Lambda},
$$

where $f_{e}=1+3 / 2 e_{1}^{2}+1 / 8 e_{1}^{4}$ and

$$
\Lambda=m_{2}^{2} k_{L, 1} R_{1}^{5}+m_{1}^{2} k_{L, 2} R_{2}^{5},
$$

where $R_{j}$ and $k_{L, j}$ are the radius and the apsidal motion constant, respectively, of the $j \in 1,2$ object in the inner binary. Equating these two timescales, and solving for $a_{1}$, we further simplify this 

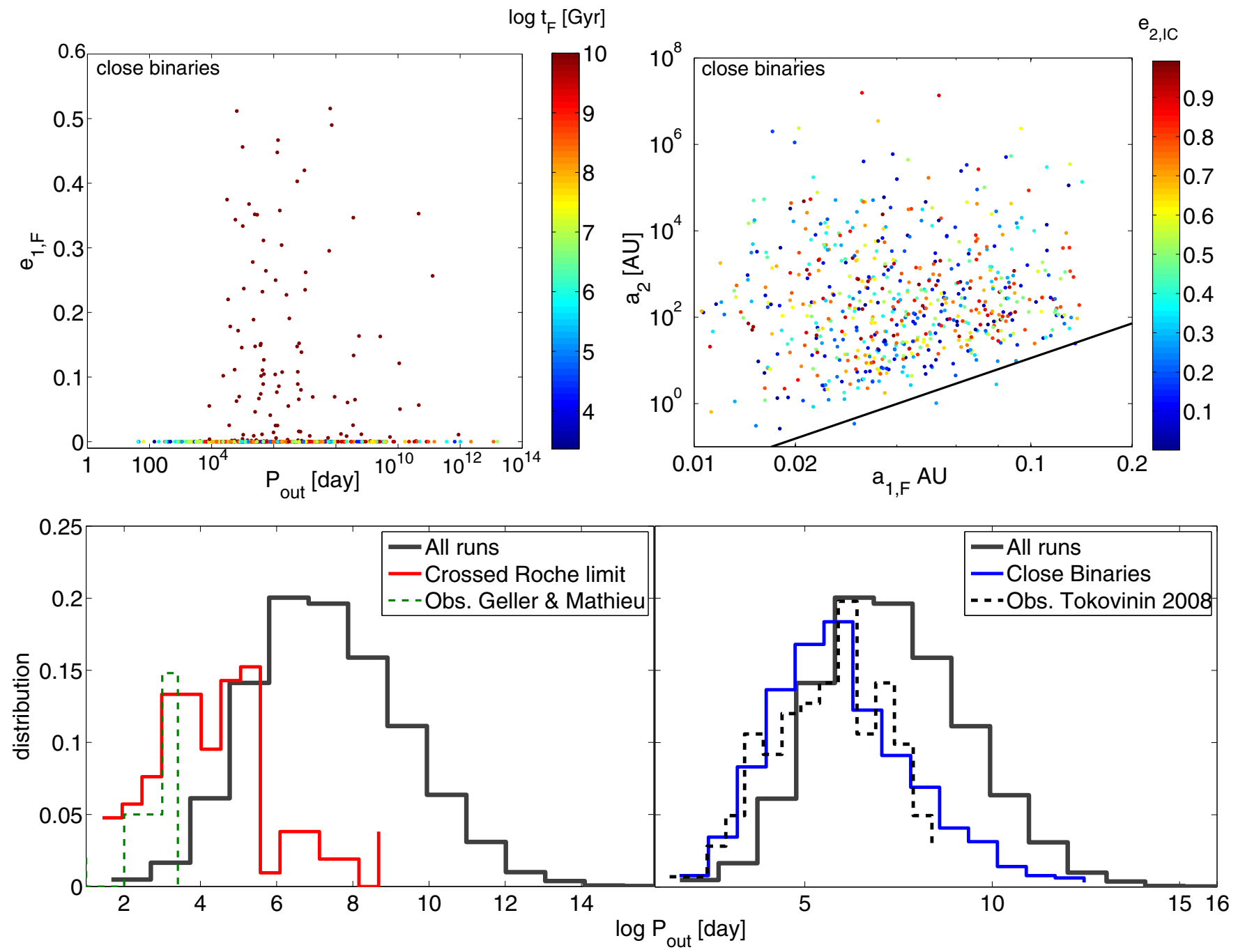

Figure 8. Outer orbit configuration. Right top panel: outer orbit separation $\left(a_{2}\right.$, which does not change during the evolution) vs. the final inner orbit separation $a_{1, F}$, for the close binary population. The different colors mark the initial outer orbital eccentricity (which is, on average, larger than 0.5). Note that the outer orbital eccentricity does not change significantly for the population of close binaries (see text). By the solid line we mark the analytical trend for similar-mass systems using Equation (9). Note that $\sim 50 \%$ of the systems (those with $a_{2} \lesssim 100 \mathrm{AU}$ ) started out with $a_{1}>0.1 \mathrm{AU}$ and have migrated to the left side of the theoretical line. Left top panel: inner orbit final eccentricity, $e_{1, F}$, as a function of the outer orbit period $P_{\text {out }}$, for the close binary population. The different colors mark the time at which the integration stopped. This shows that those systems with large final eccentricity are still undergoing tidal evolution and have systematic larger outer orbit periods $\left(\gtrsim 10^{4}\right.$ days)). Bottom panels: outer orbit period distribution. In both panels we consider all of the runs (blue-gray line). Right bottom panel: we show the period of the companion of the close binaries (blue line) and the observed distribution, scaled to match the theory lines, (Tokovinin 2008). Left bottom panel: we show the period of the merged population (red line) and the observed blue straggler distribution of NGC 188 (Geller \& Mathieu 2012), also scaled to match the theory lines.

(A color version of this figure is available in the online journal.)

by taking $m_{1} \sim m_{2}$, which gives the following relation between the two semimajor axes:

$$
a_{2}^{3} \sim a_{1}^{8} \frac{m_{3}}{m_{1}} \frac{\left(1-e_{1}^{2}\right)^{5}}{60 R_{1}^{5} k_{L, 1} f_{e}\left(1-e_{2}\right)^{3 / 2}} .
$$

This relation can be used to constrain the other parameters in the problem, for a given inner and outer separation.

We further approximate Equation (9) by taking $m_{3} \sim m_{1}$, $e_{1} \rightarrow 0.5$ (which is the mean initial distribution), and $e_{2} \rightarrow 0.5$, since most of the systems that produced close binaries had $e_{2} \sim 0.5$ (see Figure 8, top right panel). We get the limit of the relation between the final inner and outer orbit separations (solid line in the top panel of Figure 8). Considering the entire population, $a_{2}$ seems uncorrelated with the final inner orbit separation, $a_{1, F}$ (in agreement with Tokovinin 2008). However, this limiting line suggests that different masses and eccentricities will result in different relations. In Figure 8, about $50 \%$ of the systems with $a_{2} \lesssim 100 \mathrm{AU}$ (the relevant systems for this theoretical line) have started to the right side of this line (i.e., with $a_{1}$ initially larger than the final configuration).

In the top left panel of Figure 8 we show the final inner orbital eccentricity, $e_{1, F}$, versus the outer orbit period, $P_{\text {out }}$, for the close binary population. As depicted here, systems with outer orbit period below $\sim 30$ yr have a circular inner orbit. The inner binaries that are still undergoing tidal circularization even after 10 Gyr of the integration time (i.e., have nonnegligible final eccentricity) are naturally more likely to have wider outer orbits, although wide outer orbits can also cause the inner orbit to shrink and circularize.

Another interesting observable is the outer orbit distribution of the inner systems that merged, shown in the bottom left panel of Figure 8 (red line). These systems are now binaries, and thus $P_{\text {out }}$ is the "new" binary period. Again, as for $P_{\text {out }}$ of the inner 


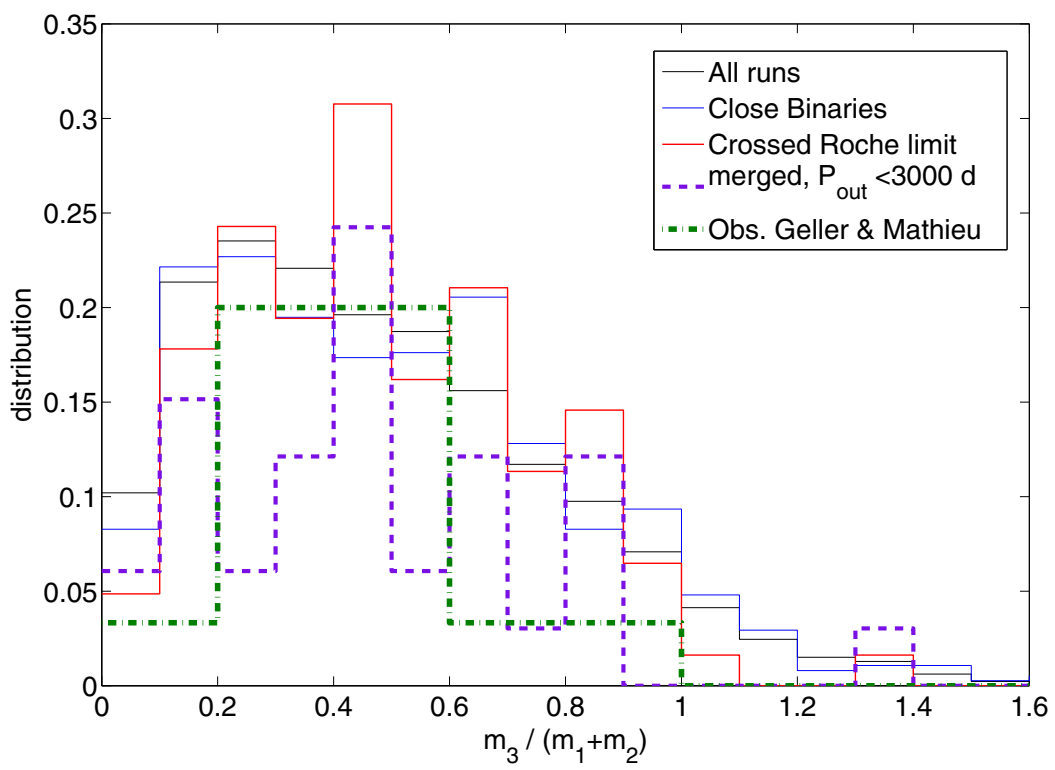

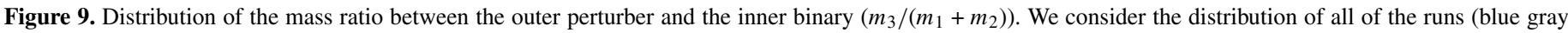

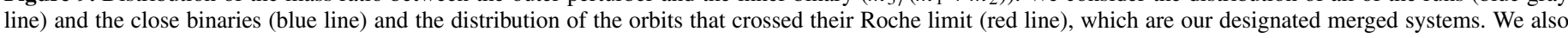

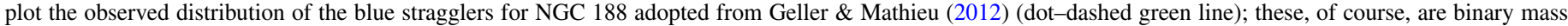

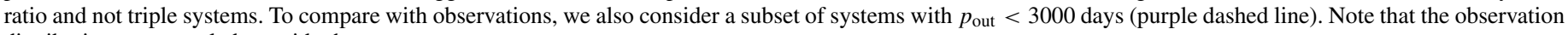
distribution was rescaled to guide the eye.

(A color version of this figure is available in the online journal.)

close binaries, the outer orbit population of those inner binaries that merged is a distinct subset of the initial period distribution. Not surprisingly, typically close outer orbits will result in a merger of the inner binaries, but as shown in this figure, a long tail of very wide outer orbit periods (up to $10^{9}$ days) can still cause the inner binaries to merge.

These merger products are thus blue stragglers. Perets \& Fabrycky (2009) suggested a similar mechanism for the formation of blue stragglers; however, they mainly envisioned a two-step process, in which three-body dynamics plus tidal dissipation created a close binary, and that binary subsequently merged by magnetic winds or had unstable or efficient mass transfer. Their mechanism explained the contemporaneous observation of a high fraction of companion stars to blue stragglers (Mathieu \& Geller 2009). However, more recently Geller \& Mathieu (2011) found secondary masses consistent with the typical mass of a white dwarf $\left(\sim 0.5 M_{\odot}\right)$, whereas in the Perets \& Fabrycky (2009) scenario and ours, the companion masses simply echo the initial conditions of triples-see Figure 9. Also, Gosnell et al. (2014) found the UV light of a white dwarf in several systems, suggesting the remnant of a mass donor, rather than the distant companion of the triple dynamics scenario.

However, we see several reasons why the case is not yet closed in favor of stable mass transfer. First, the recent simulations of Geller et al. (2013) suggest that not enough blue stragglers are made by the standard prescriptions for mass transfer in the beststudied cluster (NGC 188). Second, five blue stragglers have no companions detected out to 3000 day orbital periods (Mathieu \& Geller 2009); even if there is a more distant companion, this would be too wide for mass transfer to make a blue straggler. Third, the secondary stars typically have nonzero eccentricities, which a priori would not be expected after the red-giant phase of one of the stars (Verbunt \& Phinney 1995; although given the uncertain mass-transfer physics, it may be possible; Sepinsky et al. 2009). Our mechanism can address these three aspects.
The general principle of collisions in triples was in Geller et al. (2013)'s $N$-body model (they specifically saw Leigh \& Sills (2011)'s mechanism of collision during unstable resonant encounters), and they also followed stellar evolution models to account for the mass-transfer systems. They computed fewer blue stragglers than are actually seen in the cluster, however. We reiterate two caveats Geller et al. (2013) noted: the model lacked primordial triples, and for the dynamically formed triples it used a formalism that accounted only for the quadrupole interaction (the prescription of Mardling \& Aarseth (2001) within NBODY6). Having primordial triples may increase the yield of blue stragglers, as the outer binary may be perturbed to higher inclination or eccentricity by passing stars, which then triggers a collision by EKL evolution. And we have shown that the octupole interaction is much more efficient at generating collisions than the quadrupole alone. So we suggest that these extra components may explain the shortfall in modeled blue stragglers.

On this second point, we note that our mechanism could explain many of the detected binaries, but it also naturally produces a range of companion periods that could be longer than 3000 days. The cluster environment should unbind the longest-period blue straggler binaries. These points were also made by Geller et al. (2013).

Finally, we note that our mechanism naturally predicts an eccentric companion to blue stragglers. Actually, it appears to produce higher eccentricities than are seen in Mathieu \& Geller's (2009) observations. In the bottom panel of Figure 10 we show the final distribution of $e_{2}$ of the outer orbit companions for the merged inner systems (red line). During the EKL evolution, the outer orbital eccentricity undergoes small-amplitude oscillations ( $\mathrm{NaOz}$ et al. 2013a); however, as pointed out by Teyssandier et al. (2014) in the case of comparable-mass perturbers, large inner orbital eccentricities are reached when the outer orbital eccentricity almost does not vary. We also find that the final outer orbital eccentricity almost does not change for 


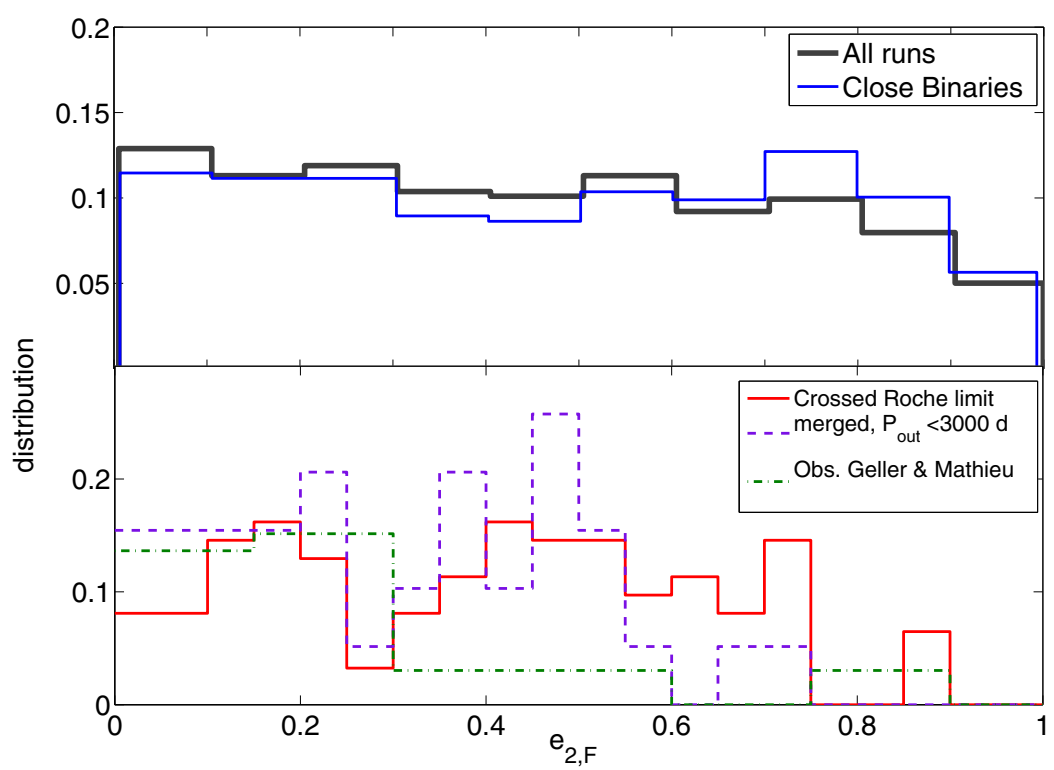

Figure 10. Distribution of the final outer orbit's eccentricity $\left(e_{2, F}\right)$. Note that the final outer orbital eccentricity almost does not change for those systems that ended up either as close binaries or as merged systems. In the top panel we consider the distribution of all of the runs (blue gray line) and the closed binaries (blue line). In the bottom panel we consider the distribution of the orbits that crossed their Roche limit (red line), which are our designated merged systems. We also plot the observed distribution of the blue stragglers for NGC 188 adopted from Geller \& Mathieu (2012) (dot-dashed green line). To compare with observations, we also consider a subset of systems with $p_{\text {out }}<3000$ days (purple dashed line). Note that the observation distribution was rescaled to guide the eye.

(A color version of this figure is available in the online journal.)

those systems that ended up either as close binaries or as merged systems. Moreover, the merged systems have a distinct $e_{2, F}$ population compared to all of the runs (top panel) that preferentially favor large eccentricities. We also overplot the observed eccentricity distribution of the blue stragglers in NGC 188, (Geller \& Mathieu 2012). This distribution is limited to blue straggler binaries with period smaller than 3000 days. Therefore, to compare with observations, we also consider a subset of systems with $P_{\text {out }}<3000$ days.

\section{DISCUSSION}

We have studied the secular evolution of triple stellar systems while considering the octupole level of approximation of the hierarchical three-body problem. During the system evolution, the octupole level of approximation can cause large eccentricity excitations for the inner orbit. During the large eccentricity excursions, tidal interactions play an important role. On close pericenter passages, when tides are important, the orbital energy is dissipated, the separation shrinks, and the orbit circularizes (see right panels of Figure 2). The final orbit stabilized on a separation that balances eccentricity excitations from the EKL mechanism and tidal (and/or GR) precession. On the other hand, if the eccentricity excursion happens on a relative short timescale (but still long so the secular approximation is valid, e.g., Naoz et al. 2013a), and tidal (or GR) force cannot influence the dynamics, the binary members may cross each other's Roche limits (see left panels of Figure 2). We considered the systems that crossed their Roche limits as merged systems.

\section{Comparison with observations.}

We found that $\sim 21 \%$ of all our runs ended up with $p_{\text {in }} \lesssim$ 16 days, and $4 \%$ of all the systems crossed the Roche limit (the merged systems). We find that the final inner orbit's period distribution agrees with the observed distribution adopted from the Tokovinin (2008) public catalog (see Figure 1). Furthermore, the inner member configurations that resulted in close binaries (merged systems) represent a distinct subset population from the initial injected binary distribution, which constrains the birth properties of close binaries (merged systems). This also suggests that these subsets may have only weak dependency on the properties of the initial injected distribution of all triples. As shown in Figure 1, the close systems had an initial inner period peak of $\sim 10$ days, while the merged systems had an initial period peak of $\sim 1000$ days. Both subsets have a long tail of wide systems that can undergo separation shrinking, ending up as either a close binary or a merged system, where wider inner binaries are slightly more likely to end up in a merged configuration than a close binary.

An interesting consequence of our results, and specifically the bimodal distribution in Figure 1, is that only relatively wide inner binaries ( $\gtrsim 16-40$ days) are available for the EKL evolution at the white dwarf stage (where mass loss will tend to widen the configuration even further). Furthermore, many of these will not be in a near-polar configuration (see Figure 5). Many of those close binaries have already decoupled from the third star (see Figure 3) and are unlikely to undergo large eccentricity excitations at a later stage. Thus, this distribution should be taken into account for the probability estimations of the Type Ia double-degenerate scenario through triple-body evolution (e.g., Thompson 2011; Prodan et al. 2013; Katz \& Dong 2012; Dong et al. 2014).

Tokovinin (2008) showed that $P_{\text {out }} / P_{\text {in }}$ have large values and concluded that the Kozai (TPQ) eccentricity excitations are suppressed due to GR precessions and that therefore this mechanism cannot produce tight binaries with a wide outer perturber. Here we claim the exact opposite and support it by qualitative comparison with observations. The EKL mechanism in the presence of tides naturally produces very tight binaries with a companion on a large range of periods (see Figures 3 and 8). When the inner orbit is longer initially, GR does not quench eccentricity excitations, and 
thus tidal dissipation can still take place. Furthermore, the EKL mechanism, compared to the TPQ case, extends the valley in the period distribution to larger values with a rather flat period distribution for the close binaries as seen in observations (see Figure 1 and discussion in Section 3). We also find that the outer orbital period distribution is consistent with observations, both for close inner binary systems (see Figure 8, bottom right panel, where we compared to the Tokovinin (2008) catalog of observed triples) and for the companion of the merged population adopted from Geller \& Mathieu (2012) (see Figure 8, bottom left panel). The strong agreement with observations for both of those populations emphasizes the notion that three-body secular interactions may be the main channel for close inner binaries and merged systems like blue stragglers. Future observations can further help test this idea.

2. The implications of the mechanism on blue straggler formation.

The two main mechanisms that have been proposed in the literature to explain the formation of blue stragglers are coalescence and mass transfer between two stars (McCrea 1964), or collision between the stellar members in a binary either in the field (Hills \& Day 1976) or induced by gravitational perturbations of a third object (Perets \& Fabrycky 2009). The latter mechanism is especially promising in explaining blue straggler binaries. Here we found that about $4 \%$ of our runs have crossed each others Roche limit. Both mechanisms may contribute; however, here we focus on the secular interactions, and specifically the EKL mechanism.

The merger between the inner orbit's members typically happens after 5-100 quadrupole timescales (Equation (6)). In the example shown in the left panel of Figure 2, the merger happened after $\sim 15$ quadrupole timescales; however, for nearly coplanar systems, the large eccentricity peak can happen after just a few quadrupole timescales ( $\mathrm{Li}$ et al. 2013). Regardless of the exact number of quadrupole timescales before a merger, the merger is not immediate. This implies that the stars typically will be already on the main-sequence phase; furthermore, the members will cross the Roche limit during a large eccentricity phase. Therefore, during the evolution we expect an electromagnetic signature that will result from the large velocities (due to the large eccentricities) of the two main-sequence stars.

Given these typical numbers, most of the systems that crossed their Roche limit did so in less than few tens of Myr. Therefore, without cluster dynamics included, this mechanism would not make blue stragglers in an open cluster with the age of a few Gyr. However, primordial triples may be torqued to higher mutual inclination and eccentricity at some point during the life of the cluster, which would then lead to EKL oscillations and a merger, and thus an observable blue straggler. Measuring the triple fraction for open clusters may support this claim that the EKL takes place for newly formed or newly perturbed triples. However, the estimations of triples from observations suffer from incompleteness and place a lower

\footnotetext{
7 Note that if this mechanism of the formation of close binaries is the dominating channel, it means that during the inner orbital shrinking test particles (such as planets) may be ejected from the system. Thus, we would expect a deficit of circumbinary planets, in agreement with observations (e.g., Armstrong et al. 2014).
}

limit that ranges from $0.5 \%$ to $2.3 \%$ for different clusters and observations (e.g., Mermilliod et al. 1992; Mermilliod \& Mayor 1999; Geller et al. 2010). N-body calculations showed that triples can be dynamically generated in the course of 7 Gyr to about $3.8 \%$ and maximum of $4.5 \%$ (Geller et al. 2013). Furthermore, comparing the type and configurations of the $\mathrm{N}$-body simulated triples to the observations led Geller et al. (2013) to conclude that open clusters may form with a significant population of primordial triples, and they are continuing to form dynamically. Thus, for our purposes, new triples are being formed throughout the lifetime for the cluster, allowing for the EKL mechanism to take place again for each new triple, which can produce blue stragglers. Thus, the blue stragglers we observe now are recently formed.

A caveat for these calculations lies in our tidal model. The exact fraction of merged systems depends on the tidal model and the timescales assumed. Naoz et al. (2012) showed that the final fraction of hot Jupiters that merged into their stars depends on the viscous time assumed in the model. Specifically, having shorter viscous timescales by two orders of magnitude compared to the nominal one resulted in zero merged systems, where two orders of magnitude longer timescales resulted in half as many merged systems. It is reasonable to assume that similar variations will occur here.

Recently Gosnell et al. (2014) reported the detection of three young white dwarf companions to blue stragglers in the NGC 188 star cluster. Interestingly, one of the binaries has a large period ( $~ 3030$ days) and could not be explained as a simple mass transfer or wind accretion binary. We suggest that this binary may be a result of the dynamical interaction discussed here. The relatively short age of the white dwarf may suggest that this system formed not too long ago. Gosnell et al. (2014) suggested that the other two binaries were formed through mass transfer and common envelope episodes and needed an almost unity mass ratio between the two members. This is rather surprising, since if these two binaries are selected randomly, their mass ratio should not be one. One possibility is that indeed blue stragglers have a unique mass function that is close to twins. The second possibility is that even the short-period detections are evidence of a dynamical origin.

\section{Implications on the obliquity distribution.}

Considering the systems that ended up in close systems ( $\sim 16$ days), we predict a specific distribution for the mutual inclination of the orbit; see Figure 5. This distribution is a specific signature for secular three-body evolution of the system. Another promising observable is the obliquity of the inner binaries. We found that the final obliquity distribution has a signature of the initial properties (see Figure 6), which can be used as a tool to study the formation conditions of close binaries in triples. Thus, the obliquity distribution has an aligned peak. Furthermore, we suggested that observed misaligned binaries such as CV Velorum (Albrecht et al. 2014) and DI Herculis (Albrecht et al. 2009) may have a perturber on a wide orbit, as their current period and obliquity values are consistent with the predicted obliquity distribution of our simulated triple systems.

We have run three Monte Carlo runs, which differ by the initial obliquities of the inner binary. We found that most of the closest binaries have aligned configurations, while the wider ones have a broad obliquity distribution that results 
from the initial condition. This is most apparent for the Monte Carlo test that was set initially with a perpendicular configuration. This final distribution, for intermediate to long periods, is consistent with a broad distribution with a clear peak at $90^{\circ}$; see the bottom right panel of Figure 6 . Thus, obtaining a large sample of observed obliquity distributions can help shed light on the formation properties of those systems.

We thank Aaron Geller for useful discussions, suggestions, and answering many questions. We also thank Tassos Fragos, Ramesh Narayan, Amaury Triaud, Todd Thompson, and Simon Albrecht for useful discussions. We also thank Andrei Tokovinin for discussing our arguments about the comparison between theory and observations. In addition, we thank our anonymous referee for a thorough reading of the paper and providing many useful comments and suggestions. S.N. is supported by NASA through an Einstein Postdoctoral Fellowship awarded by the Chandra X-ray Center, which is operated by the Smithsonian Astrophysical Observatory for NASA under contract PF2-130096.

\section{REFERENCES}

Albrecht, S., Reffert, S., Snellen, I. A. G., \& Winn, J. N. 2009, Natur, 461, 373 Albrecht, S., Setiawan, J., Torres, G., Fabrycky, D. C., \& Winn, J. N. 2013, ApJ, 767,32

Albrecht, S., Winn, J. N., Carter, J. A., Snellen, I. A. G., \& de Mooij, E. J. W. 2011, ApJ, 726, 68

Albrecht, S., Winn, J. N., Fabrycky, D. C., Torres, G., \& Setiawan, J. 2012, in IAU Symp. 282, From Interacting Binaries to Exoplanets: Essential Modeling Tools, ed. M. T. Richards \& I. Hubeny (Cambridge: Cambridge Univ. Press), 397

Albrecht, S., Winn, J. N., Torres, G., et al. 2014, ApJ, 785, 83

Antognini, J. M., Shappee, B. J., Thompson, T. A., \& Amaro-Seoane, P. 2014, MNRAS, 439, 1079

Antonini, F., Murray, N., \& Mikkola, S. 2014, ApJ, 781, 45

Armstrong, D. J., Osborn, H., Brown, D., et al. 2014, MNRAS, 444, 1873

Blaes, O., Lee, M. H., \& Socrates, A. 2002, ApJ, 578, 775

Bode, J. N., \& Wegg, C. 2014, MNRAS, 438, 573

Conroy, K. E., Prsa, A., Stassun, K. G., et al. 2014, AJ, 147, 45

Dong, S., Katz, B., Kushnir, D., \& Prieto, J. L. 2014, arXiv:1401.3347

Duquennoy, A., \& Mayor, M. 1991, A\&A, 248, 485

Eggleton, P. P. 1983, ApJ, 268, 368

Eggleton, P. P., Kiseleva, L. G., \& Hut, P. 1998, ApJ, 499, 853

Eggleton, P. P., \& Kiseleva-Eggleton, L. 2001, ApJ, 562, 1012

Eggleton, P. P., \& Kisseleva-Eggleton, L. 2006, Ap\&SS, 304, 75

Eggleton, P. P., Kisseleva-Eggleton, L., \& Dearborn, X. 2007, in IAU Symp. 240, Binary Stars as Critical Tools \& Tests in Contemporary Astrophysics, ed. W. I. Hartkopf, E. F. Guinan, \& P. Harmanec (Cambridge: Cambridge Univ. Press), 347

Fabrycky, D., \& Tremaine, S. 2007, ApJ, 669, 1298

Fabrycky, D. C., Johnson, E. T., \& Goodman, J. 2007, ApJ, 665, 754

Ford, E. B., Kozinsky, B., \& Rasio, F. A. 2000, ApJ, 535, 385

Geller, A. M., Hurley, J. R., \& Mathieu, R. D. 2010, in IAU Symp. 266, Star Clusters: Basic Galactic Building Blocks Throughout Time and Space, ed. R. de Grijs \& J. R. D. Lépine (Cambridge: Cambridge Univ. Press), 258

Geller, A. M., Hurley, J. R., \& Mathieu, R. D. 2011, BAAS, 43, 327.02

Geller, A. M., Hurley, J. R., \& Mathieu, R. D. 2013, AJ, 145, 8

Geller, A. M., \& Mathieu, R. D. 2011, Natur, 478, 356

Geller, A. M., \& Mathieu, R. D. 2012, AJ, 144, 54
Goldreich, P., \& Soter, S. 1966, Icar, 5, 375

Gosnell, N. M., Mathieu, R. D., Geller, A. M., et al. 2014, ApJL, 783, L8 Griffin, R. F. 2012, JApA, 33, 29

Hamers, A. S., Pols, O. R., Claeys, J. S. W., \& Nelemans, G. 2013, MNRAS, 430, 2262

Hansen, B. M. S. 2010, ApJ, 723, 285

Harding, L. K., Hallinan, G., Konopacky, Q. M., et al. 2013, A\&A, 554, A113 Harrington, R. S. 1968, AJ, 73, 190

Harrington, R. S. 1969, CeMec, 1, 200

Hills, J. G., \& Day, C. A. 1976, ApL, 17, 87

Holman, M., Touma, J., \& Tremaine, S. 1997, Natur, 386, 254

Hut, P. 1980, A\&A, 92, 167

Ivanova, N., Chaichenets, S., Fregeau, J., et al. 2010, ApJ, 717, 948

Katz, B., \& Dong, S. 2012, arXiv:1211.4584

Katz, B., Dong, S., \& Malhotra, R. 2011, PhRvL, 107, 181101

Kiseleva, L. G., Eggleton, P. P., \& Mikkola, S. 1998, MNRAS, 300, 292

Kozai, Y. 1962, AJ, 67, 591

Kushnir, D., Katz, B., Dong, S., Livne, E., \& Fernández, R. 2013, ApJL, 778, L37

Leigh, N., \& Sills, A. 2011, MNRAS, 410, 2370

Leigh, N. W. C., \& Geller, A. M. 2013, MNRAS, 432, 2474

Levrard, B., Correia, A. C. M., Chabrier, G., et al. 2007, A\&A, 462, L5

Li, G., Naoz, S., Holman, M., \& Loeb, A. 2014, ApJ, 791, 86

Li, G., Naoz, S., Kocsis, B., \& Loeb, A. 2013, ApJ, 785, 116

Lidov, M. L. 1962, P\&SS, 9, 719

Lithwick, Y., \& Naoz, S. 2011, ApJ, 742, 94

Mardling, R. A., \& Aarseth, S. J. 2001, MNRAS, 321, 398

Mathieu, R. D., \& Geller, A. M. 2009, Natur, 462, 1032

Mazeh, T., \& Shaham, J. 1979, A\&A, 77, 145

McCrea, W. H. 1964, MNRAS, 128, 147

Mermilliod, J.-C., \& Mayor, M. 1999, A\&A, 352, 479

Mermilliod, J.-C., Rosvick, J. M., Duquennoy, A., \& Mayor, M. 1992, A\&A, 265,513

Michaely, E., \& Perets, H. B. 2014, arXiv:1406.3035

Miller, M. C., \& Hamilton, D. P. 2002, ApJ, 576, 894

Naoz, S., Farr, W. M., Lithwick, Y., Rasio, F. A., \& Teyssandier, J. 2011, Natur, 473, 187

Naoz, S., Farr, W. M., Lithwick, Y., Rasio, F. A., \& Teyssandier, J. 2013a, MNRAS, 431, 2155

Naoz, S., Farr, W. M., \& Rasio, F. A. 2012, ApJL, 754, L36

Naoz, S., Kocsis, B., Loeb, A., \& Yunes, N. 2013b, ApJ, 773, 187

Pejcha, O., Antognini, J. M., Shappee, B. J., \& Thompson, T. A. 2013, MNRAS, 435, 943

Perets, H. B. 2014, arXiv: 1406.3490

Perets, H. B., \& Fabrycky, D. C. 2009, ApJ, 697, 1048

Perets, H. B., \& Kratter, K. M. 2012, arXiv:1203.2914

Pribulla, T., \& Rucinski, S. M. 2006, AJ, 131, 2986

Prodan, S., Murray, N., \& Thompson, T. A. 2013, arXiv:1305.2191

Raghavan, D., McAlister, H. A., Henry, T. J., et al. 2010, ApJS, 190, 1

Rappaport, S., Deck, K., Levine, A., et al. 2013, ApJ, 768, 33

Sepinsky, J. F., Willems, B., Kalogera, V., \& Rasio, F. A. 2009, ApJ, 702,1387

Shappee, B. J., \& Thompson, T. A. 2013, ApJ, 766, 64

Teyssandier, J., Naoz, S., Lizarraga, I., \& Rasio, F. 2013, ApJ, 779, 166

Thompson, T. A. 2011, ApJ, 741, 82

Tokovinin, A. 2008, MNRAS, 389, 925

Tokovinin, A. 2014a, AJ, 147, 86

Tokovinin, A. 2014b, AJ, 147, 87

Tokovinin, A., Thomas, S., Sterzik, M., \& Udry, S. 2006, A\&A, 450, 681

Tokovinin, A. A. 1997, AstL, 23, 727

Tokovinin, A. A., \& Smekhov, M. G. 2002, A\&A, 382, 118

Triaud, A. H. M. J., Hebb, L., Anderson, D. R., et al. 2013, A\&A, 549, A18

Veras, D., \& Tout, C. A. 2012, MNRAS, 422, 1648

Verbunt, F., \& Phinney, E. S. 1995, A\&A, 296, 709

Wen, L. 2003, ApJ, 598, 419

Zhou, G., \& Huang, C. X. 2013, arXiv:1307.2249 